DEPÓSITO LEGAL ZU2020000153

Esta publicación científica en formato digital

es continuidad de la revista impresa

ISSN 0041-8811

E-ISSN 2665-0428

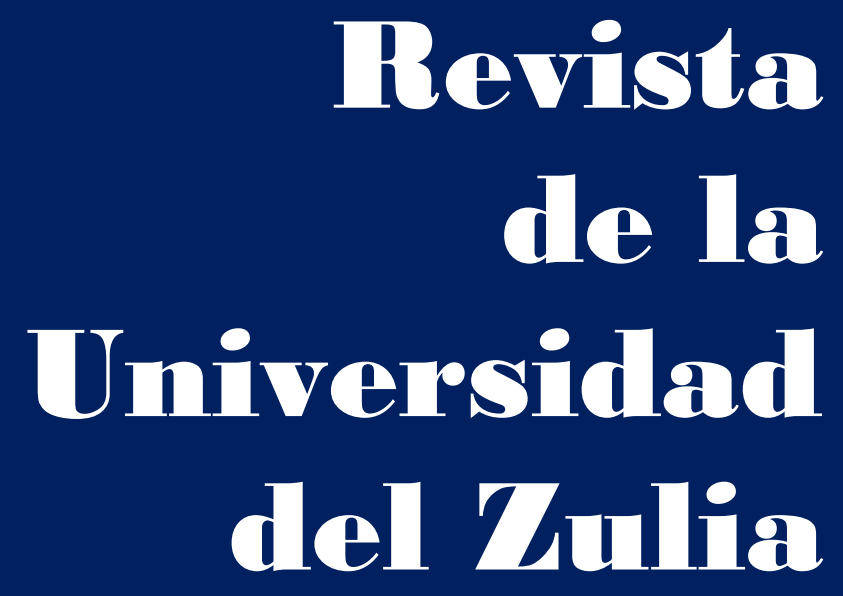

Fundada en 1947

por el Dr. Jesús Emrique Lossada

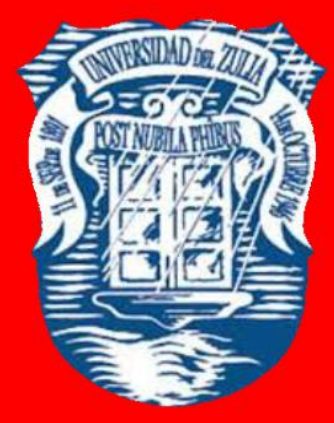

Ciencias

Sociales

y Arte

Año $12 \quad N^{\circ} 34$

Septiembre - Diciembre 2021

Tercera Época

Maracaibo-Veneruela 


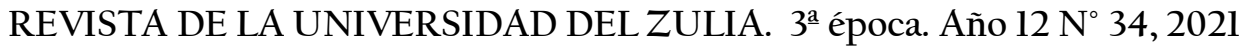

Eglee Vargas-Acosta et al. /// Cuarentena social en el contexto del COVID-19: una mirada... 442-468

DOI: http://dx.doi.org/10.46925//rdluz.34.25

\title{
Cuarentena social en el contexto del COVID-19: Una mirada desde la perspectiva de docentes universitarias eméritas
}

\author{
Eglee Vargas-Acosta * \\ Rita Navas Perozo ** \\ Mairely Nuváez de De Armas ***
}

RESUMEN

El objetivo de la investigación fue describir los significados construidos por cinco docentes universitarias eméritas de la Universidad del Zulia en Maracaibo-Venezuela, respecto a la experiencia vivida con motivo de la cuarentena social, en el contexto de la pandemia por COVID-19. Se trata de una investigación cualitativa, desde la perspectiva fenomenológica. Los hallazgos se centran en dos temas: el primero referido al contexto de la cuarentena social en el país; y el segundo, a las experiencias vividas durante esta. Emergieron dos categorías: La cuarentena en Venezuela, es diferente a la de otros países; y la afectación de la cuarentena social. Se concluye que la categoría central emergente "La cuarentena en Venezuela, es diferente a la de otros países" en el contexto de la pandemia por COVID-19, resultó de interés conceptual para la comprensión del fenómeno estudiado, ya que reflejó la superposición de dos condiciones: la crisis humanitaria compleja y la pandemia misma; por lo que la cuarentena social tuvo un impacto multidimensional negativo, que afectó además de las ya deterioradas condiciones materiales de vida, también lo socio-emocional, y valores como la libertad, la paz, la convivencia intrafamiliar, todo ello con base a los significados construidos por las informantes clave.

PALABRAS CLAVE: pandemia; COVID-19; personal académico docente; Venezuela.

*Docente. Escuela de Trabajo Social. Facultad de Ciencias Jurídicas y Políticas. Universidad del Zulia (LUZ). Maracaibo-Venezuela. ORCID: https://orcid.org/0000-0001-5091-5274. E-mail: egleevargasacosta@gmail.com

**Docente. Instituto de Investigaciones. Facultad de Odontología. Universidad del Zulia (LUZ). Maracaibo-Venezuela. ORCID: https://orcid.org/0000-0002-1224-3734. E-mail: ritanavasperozo@gmail.com

***Docente. Escuela de Trabajo Social. Facultad de Ciencias Jurídicas y Políticas. Universidad del Zulia (LUZ). Maracaibo-Venezuela. ORCID: https://orcid.org/0000-0001-5785-9447. E-mail: mairelynuvaez@hotmail.com

Recibido: 25/06/2021

Aceptado: 12/08/2021 


\section{Social quarantine in the context of COVID-19: A look from the perspective of emeritus university professors}

ABSTRACT

The objective of the research was to describe the meanings constructed by five emeritus university professors from the University of Zulia in Maracaibo-Venezuela, regarding the experience lived on the occasion of the social quarantine, in the context of the COVID-19 pandemic. It is a qualitative investigation, from a phenomenological perspective. The findings focus on two issues: the first refers to the context of social quarantine in the country; and the second, to the experiences lived during this. Two categories emerged: The quarantine in Venezuela is different from that of other countries; and the impact of social quarantine. It is concluded that the emerging central category "The quarantine in Venezuela is different from that of other countries" in the context of the COVID-19 pandemic, was of conceptual interest for understanding the phenomenon studied, since it reflected the overlap of two conditions: the complex humanitarian crisis and the pandemic itself; Therefore, the social quarantine had a negative multidimensional impact, which affected, in addition to the already deteriorated material living conditions, also the socio-emotional, and values such as freedom, peace, family coexistence, all based on the meanings constructed by key informants.

KEYWORDS: Pandemics; COVID-19; academic teaching personnel; Venezuela.

\section{Introducción}

Actualmente en el mundo entero, aun se viven momentos de inquietud e incertidumbre con respecto a la pandemia producto del virus Sars-Cov-2, responsable de la enfermedad COVID-19 (por sus siglas en inglés). A finales de febrero 2020, las cifras alcanzadas de personas contagiadas, condujo a que la mayoría de los países del mundo tomaran iniciativas estratégicas para neutralizar el contagio masivo, es decir, intentar frenar su propagación y su alto impacto negativo tanto en los miembros de la sociedad, como en la estructura del sistema de salud de los respectivos países, debido a la demanda por los altos porcentajes de infectados.

Cabe destacar que el descubrimiento de los coronavirus, data de la década del 60, como un tipo de virus en cuya imagen se visualiza una corona a su alrededor; sin embargo, este "nuevo coronavirus es una cepa particular que no había sido identificada previamente en 
REVISTA DE LA UNIVERSIDAD DEL ZULIA. 3a época. Año $12 \mathrm{~N}^{\circ} 34,2021$ Eglee Vargas-Acosta et al. /// Cuarentena social en el contexto del COVID-19: una mirada... 442-468 DOI: http://dx.doi.org/10.46925//rdluz.34.25

humanos" (OMS, 2020). Al principio, se señaló que el contagio era por medio de los animales como fuente de infección humana; luego por el contacto físico, después que también podía ser por vía aérea; a pesar de esto, para ese momento se destacó que "existe muy poca información sobre la transmisión, gravedad e impacto clínico, con solo unos pocos casos notificados hasta el momento" (OMS, 2020b). Lo que sí ha estado claramente precisado es que, sin lugar a dudas, el COVID-19 es una enfermedad de alto contagio que surgió en Wuhan - China y ha alcanzado proporciones globales desde que fue reconocido por parte de la OMS.

La OMS (2021), sigue advirtiendo entre los síntomas más frecuentes del COVID-19: fiebre, dolor de cabeza, tos y cansancio; entre los menos frecuentes: dolores y molestias, dolor de garganta, diarrea, conjuntivitis, pérdida del gusto o el olfato, sarpullido en la piel o decoloración de los dedos de las manos o pies: y, entre los graves: dificultad para respirar o disnea, dolor u opresión en el pecho y la pérdida del habla o del movimiento. Es importante destacar los casos de personas que aun dando positivo para el virus en las pruebas del laboratorio, no presentaron ningún síntoma, lo que es conocido como una infección asintomática. Al inicio se reportaba que atacaba y podía generar situaciones de salud más delicadas, principalmente a la población de alto riesgo o vulnerables como personas mayores, personas inmunocomprometidas, personas inmunodepresivas o con afecciones crónicas, tales como: diabetes mellitus, insuficiencia renal, cáncer, o neumopatía crónica (OMS, 2020).

En este contexto, esta organización definió cuatro situaciones de la transmisión: ausencia de casos, casos esporádicos, conglomerados de casos y transmisión comunitaria, y enfatizó que "a medida que evolucione la pandemia, los países o zonas pasarán de una situación de transmisión a otra (en cualquiera de las direcciones) y podrán experimentar rebrotes de casos intercalados con períodos con un nivel bajo de transmisión" (OMS, 2020).

Diversas han sido las situaciones de incertidumbre y efectos generados en las personas y grupos familiares, con motivo de la cuarentena social como la estrategia para evitar el contagio masivo del mencionado virus e incluso en las organizaciones e instituciones de educación en todos los subsistemas. A ello hay que sumarle situaciones de vulnerabilidad en países en desarrollo que evidencian desigualdades sociales en diversas áreas, tales como: educación, culturales, empleo, y salud, entre otros. 
REVISTA DE LA UNIVERSIDAD DEL ZULIA. 3a época. Año $12 \mathrm{~N}^{\circ} 34,2021$ Eglee Vargas-Acosta et al. /// Cuarentena social en el contexto del COVID-19: una mirada... 442-468 DOI: http://dx.doi.org/10.46925//rdluz.34.25

Venezuela se ha caracterizado en las últimas décadas por un profundo estatismo, endeudamiento externo, devaluación de la moneda, incremento de la importación de productos y una ampliación del mercado del trabajo informal (Carrillo, 2018); así como altos índices inflacionarios, que según cifras oficiales del Banco Central de Venezuela (BCV) solo en el acumulado de los meses de enero a julio de 2020, la inflación fue de 184,5; estadísticas no oficiales reportan cifras cercanas al 10.000\% en el 2019 (Daniels, 2020). Lo que condujo a la declarativa de "Emergencia Humanitaria Compleja" (Richter y Stephany, 2021), de tal manera que en la situación de la pandemia por COVID-19, los países con características y condiciones económicas y sociales frágiles como las descritas, tendrían un impacto negativo aún mayor en la calidad de vida de su población, profundizando las inequidades sociales.

De tal manera que, Venezuela en el contexto de la pandemia por covid-19, se encontraría en una situación de mayor vulnerabilidad como país que vive una crisis humanitaria, que se expresa en un elevado nivel de pobreza, condiciones insalubres, con una población infantil y adulta en estado de malnutrición y, sistemas de salud deficientes y fragmentados (Serrano, 2021), por lo que la pandemia se superpone a una crisis ya existente que ha afectado aún más el estado de deterioro no solo de la salud, sino la alimentación y nutrición de la población "en un marco de continuo detrimento social, económico, institucional y político, que no contribuye a mejorar el bienestar de las personas" (Landaeta et al. cp. Herrera-Cuenca, 2021)

Ante esta compleja trama social, se evidencia la necesidad de esta investigación, que no solo describe si no que intenta aproximarse a la comprensión y explicación de la realidad vivida por un sector de la sociedad como lo es el docente universitario emérito y adulto mayor, que vive en unas condiciones socio-económicas que se expresan en el deterioro del poder adquisitivo para satisfacer las necesidades básicas esenciales, bajas remuneraciones económicas, pérdida de la previsión y seguridad social, entre otros, conduciéndoles a una movilidad social descendente (Comisión Económica para América Latina y el Caribe, 2020), hacia niveles casi de subsistencia, al punto de impactar su calidad de vida; situación que no está alejada ni es diferente a la que viven otros profesionales y el resto de los miembros de la sociedad venezolana. 
REVISTA DE LA UNIVERSIDAD DEL ZULIA. 3ㄹe época. Año 12 N$^{\circ}$ 34, 2021 Eglee Vargas-Acosta et al. /// Cuarentena social en el contexto del COVID-19: una mirada... 442-468 DOI: http://dx.doi.org/10.46925//rdluz.34.25

El objetivo de la investigación fue describir los significados construidos por cinco docentes universitarias eméritas de la Universidad del Zulia, en Maracaibo-Venezuela, respecto a la experiencia vivida en la cuarentena social, en el contexto de la pandemia por COVID-19.

1. Estrategias de salud pública para minimizar el contagio

La OMS (2020), estableció para todos los países algunas estrategias de salud pública y social como medidas de contención o prevención de la propagación de la enfermedad contagiosa. Es necesario precisar algunas diferencias y distinciones sobre estas. Se tiene que:

La cuarentena, tiene como objetivo prevenir o evitar la propagación de la enfermedad en el momento en que las personas empiezan a presentar síntomas. Consiste en la separación e implementación de algunas restricciones del desplazamiento y las actividades -por un tiempo determinado- de las personas que no están enfermas pero que están en riesgo de un potencial contagio (Sánchez-Villena y De La Fuente-Figuerola, 2020; Hawryluck, Robinson, Galea, citado por: Broche-Pérez et al, 2020), en este caso, pueden haber estado expuestas al COVID-19. El establecimiento de esta efectiva estrategia es de estricto cumplimiento, por parte de las personas que deben estar o permanecer en un determinado espacio-lugar en tanto el período de incubación de la enfermedad sea superado y poder proteger la vida de éstas (Broche-Pérez, et al, 2020). Estas restricciones se aplican cuando existe la sospecha que la persona o un grupo de personas están infectadas; en estos casos se debe garantizar, no solo la asistencia médica, sino el apoyo psicológico, un espacio como refugio y la alimentación.

El aislamiento social, tiene como finalidad prevenir la propagación de la enfermedad. Se debe establecer una vez que el contagio se ha confirmado; lo que implica "la separación física de las personas contagiadas de aquellas que están sanas" (Sánchez-Villena, et al, 2020). Se sugiere para una mayor efectividad, cuando la detección de la enfermedad se realiza tempranamente y la persona que ha sido infectada se aísla a un espacio determinado para poder evitar que pueda tener contacto con otros. Para Hawryluck et al (citado por Broche-Pérez, et al, 2020) depende de la voluntad de las personas. 
REVISTA DE LA UNIVERSIDAD DEL ZULIA. 3a época. Año $12 \mathrm{~N}^{\circ} 34,2021$ Eglee Vargas-Acosta et al. /// Cuarentena social en el contexto del COVID-19: una mirada... 442-468 DOI: http://dx.doi.org/10.46925//rdluz.34.25

Un aspecto fundamental que se debe tener en cuenta en la implementación de estas dos medidas -cuarentena y aislamiento-, es el costo psicológico en las personas, el cual puede ser a corto, mediano o largo plazo y puede observarse en el comportamiento frente de las personas en situación normal (Dong, citado por Broche-Pérez, et al, 2020). Este autor, enfatiza la necesidad de monitorear las reacciones psicológicas cuyo origen estén vinculadas tanto a la cuarentena social como al aislamiento social en el contexto del COVID-19. Sugiere atenuar el impacto negativo en la salud mental, mediante el diseño y ejecución de intervenciones especialmente dirigidos a la población vulnerable (niños, niñas y adolescentes, adultos mayores, entre otros) así como el reforzamiento al sistema de salud en general y, en especial a los de salud mental.

En cuanto al distanciamiento social o físico, su propósito es la separación y mantenimiento de un espacio físico determinado entre las personas; esto implica tanto la restricción de la interacción entre las personas, es decir, se toma una determinada distancia física -al menos un metro- para evitar el contacto directo entre ellas como el alejamiento de aquellos lugares concurridos. En todo caso, es una medida general -que todas las personas deberían adoptar-, que se sugiere en una comunidad específica cuando tienen personas infectadas, -que al no estar identificadas ni aisladas-, pueden transmitir la enfermedad. Cuando se establece este concepto como estrategia, conduce al cierre de lugares que convoca a mayor concentración de personas, como lo son: las universidades, colegios, la realización de eventos sociales, deportivos, artísticos, centros comerciales, oficinas, entre otros.

Con respecto al confinamiento, es una medida estratégica de intervención comunitaria, para minimizar o reducir las interacciones sociales; la cual implica "permanecer refugiado el mayor tiempo posible, bajo nuevas normas socialmente restrictivas" (Sánchez-Villena, et al, 2020). Estos autores señalan que, en este caso, se pueden combinar con otras estrategias preventivas, tales como el distanciamiento social, el uso de tapabocas o mascarillas de manera obligatoria, la restricción de los horarios de circulación, la suspensión del transporte público, así como el cierre de las fronteras. Como estrategia, se debe aplicar cuando las anteriores no han sido lo suficientemente efectivas en la contención del contagio de una determinada enfermedad. 
REVISTA DE LA UNIVERSIDAD DEL ZULIA. 3a época. Año $12 \mathrm{~N}^{\circ} 34,2021$ Eglee Vargas-Acosta et al. /// Cuarentena social en el contexto del COVID-19: una mirada... 442-468 DOI: http://dx.doi.org/10.46925//rdluz.34.25

Además de las señaladas, es importante mencionar otro concepto involucrado, como el de concentraciones multitudinarias en el contexto del COVID-19, que se entiende como "un evento con una gran densidad de personas, en un lugar específico durante un periodo determinado de tiempo, que puede amplificar la transmisión de la COVID-19 y suponer una presión adicional para el sistema de salud de un país" (OMS, 2020). Comprende actividades recreativas, religiosas, deportivas, económicas, entre otras.

De manera que, cada país en su momento asumió algunas de las referidas estrategias de contención con base en las orientaciones de la OMS y la situación epidemiológica particular que han experimentado con respecto al COVID-19: el cierre de los planteles o centro educativos de los distintos niveles de educación, restricciones en cuanto a la circulación de las personas en las calles, la protección por medio de mascarillas y tapabocas, para evitar el contacto cercano sin protecciones con personas externas al grupo familiar al ir a los supermercados a realizar las compras; la promoción de medidas higiénicas en general y, en especial la del lavado de las manos, el cierre del comercio, es decir, todo aquello diferente al comercio de alimentos y medicinas, así como la suspensión de los eventos artístico, deportivo, religioso, social, entre otros (OMS, 2020a).

A nivel mundial, para el mes de julio de 2021, se habían notificado 188.655 .968 casos confirmados y 4.067.517 defunciones (OMS,2021). Para la región de las Américas se reportaron 74.576.502 casos y 1.957.044 muertes. En América Latina las estadísticas reportadas para ese mismo mes por la Organización Panamericana de la salud (OPS,2021), en algunos países fueron las siguientes: Brasil 19.262.512 casos confirmados, con 538.942 muertes; Argentina 4.719.952 y 100.695 defunciones; Colombia 4.583.442 y 114.833 fallecidos; Perú 2.088.143 y 194.845 muertes; Ecuador con 473.647 y 21.899 fallecidos; y en Venezuela los casos reportados ascienden a 289.362 y 3339 muertes.

Para julio 2021, el presidente de la OMS, enfatizó que se está viviendo un momento muy peligroso, debido a que se ha detectado en 98 países la rápida propagación dominante de la variante Delta -que puede seguir mutando- del coronavirus causante de la COVID-19, la cual ha sido detectada en la India; así mismo advierte por un lado, que no existe país que esté a salvo de ésta; y, por el otro, que "en países con bajas tasas de vacunación sigue habiendo 
REVISTA DE LA UNIVERSIDAD DEL ZULIA. 3a época. Año $12 \mathrm{~N}^{\circ} 34,2021$ Eglee Vargas-Acosta et al. /// Cuarentena social en el contexto del COVID-19: una mirada... 442-468 DOI: http://dx.doi.org/10.46925//rdluz.34.25

terribles escenas de hospitales saturados" (ONU, 2021; SWI, 2021). Otro aspecto importante es que se ratifica la necesidad de un seguimiento y ajuste de las medidas sanitarias y sociales de prevención, tales como: el aislamiento o cuarentena, distanciamiento social, la detección temprana de los casos, la atención clínica-hospitalaria, el uso de las mascarillas, lograr una buena ventilación en zonas cerradas, entre otros. Otra forma de afrontar consiste en "compartir equitativamente entre los países los equipos de protección, el oxígeno, las pruebas, los tratamientos y las vacunas" (ONU, 2021)

\section{Venezuela en el contexto de la pandemia}

Venezuela no escapa de toda esta situación mundial vinculada a la pandemia, por lo que el gobierno decretó en la segunda quincena del mes de marzo del 2020 "la cuarentena" como una medida para evitar el contagio masivo y así intentar detener el colapso de los servicios de salud, que para el momento presentaban un franco deterioro, en cuanto a cualquier situación de emergencia que pudiera presentarse como consecuencia de la pandemia. De manera que, el 13/03/2020 se confirmaron en el país los dos primeros casos de COVID-19, por lo que el presidente Nicolás Maduro decretó el Estado de Alarma (figura de Estado de Excepción contemplada en la Constitución Nacional, art. 337), en la que se ampara la cuarentena social "tras confirmarse que el virus que causa la gripe y neumonía COVID-19 comenzó a circular en el país" (Altuve, 2020), sumado a la confirmación de casos en Brasil y Colombia -países fronterizos-, lo cual representó un alto riesgo para Venezuela, fundamentalmente por las condiciones sociales y económicas de éste (Vargas-Acosta, 2018).

Cabe destacar que el gobierno agregó el confinamiento colectivo como prevención y contención más efectiva para detener el avance del COVID-19, y se reportó el cumplimiento de ésta en un 90\% (Comisión Presidencial, cp., Altuve, 2020). Otras medidas preventivas establecidas para evitar el contagio, recomendadas por la OMS (2020), han sido el lavado continuo y sistemático de las manos al llegar de la calle y, si no es posible realizarlo de forma continua, usar un desinfectante a base de alcohol al 70\%, no mostrar manifestaciones de afectos como el beso y abrazos; la manipulación de los alimentos de manera adecuada, para evitar su contaminación; y consumir alimentos pasteurizados y cocidos, entre otros. 
REVISTA DE LA UNIVERSIDAD DEL ZULIA. 3a época. Año $12 \mathrm{~N}^{\circ} 34,2021$ Eglee Vargas-Acosta et al. /// Cuarentena social en el contexto del COVID-19: una mirada... 442-468 DOI: http://dx.doi.org/10.46925//rdluz.34.25

En este contexto, se suscitan inquietudes respecto a las afectaciones que puedan generarse no solo en lo que concierne al contagio de la enfermedad, sino también otras áreas que con el establecimiento de la cuarentena social pueda impactarse, como por ejemplo la salud emocional, física y en las condiciones económicas de los miembros de la sociedad venezolana, especialmente porque "los períodos de confinamiento relacionados con epidemias han sido vinculados con impactos negativos en la salud mental en los adultos (mayor riesgo de trastornos emocionales, síntomas depresivos, irritabilidad, estrés" (Ron y Cuéllar-Flores, 2020), planteamiento que es ratificado por la Organización Panamericana de la Salud (OPS, 2020), cuando señala que en esta coyuntura es normal y comprensible que las personas puedan experimentar preocupación, estrés y miedo por la situación de incertidumbre y, aún más, si se toma en cuenta la situación de crisis social y económica por la que atraviesa Venezuela en los últimos años (Vargas-Acosta, 2018).

\section{Metodología}

Esta investigación se realizó con un abordaje cualitativo, desde la perspectiva fenomenológica, cuya esencia es la experiencia compartida, desde la perspectiva de las informantes clave, para describir y comprender "lo que los individuos tienen en común de acuerdo con sus experiencias con un determinado fenómeno (categorías que comparten en relación a éste)" (Creswell, citado por: Hernández, et al., 2018), con respecto a la experiencia vivida en el contexto de la cuarentena por el COVID-19 en Venezuela.

La fenomenología se concibe como "el estudio de los fenómenos tal como son experimentados, vividos y percibidos por el hombre" (Martínez, 2006:137); de allí que se caracteriza fundamentalmente por centrarse en la experiencia de las personas que "están vinculados con su mundo y pone el énfasis en su experiencia vivida, la cual aparece en el contexto de las relaciones con objetos, personas, sucesos y situaciones" (Álvarez-Gayou, 2005:86), es decir que el eje central de la fenomenología es la experiencia desde la referencia del sujeto, y contextualizada por las relaciones con las otras personas, con los sucesos y situaciones que viven y experimentan. 
REVISTA DE LA UNIVERSIDAD DEL ZULIA. 3a época. Año $12 \mathrm{~N}^{\circ} 34,2021$ Eglee Vargas-Acosta et al. /// Cuarentena social en el contexto del COVID-19: una mirada... 442-468 DOI: http://dx.doi.org/10.46925//rdluz.34.25

Las participantes de este estudio fueron docentes universitarias eméritas de la Universidad del Zulia, en Maracaibo estado Zulia-Venezuela; adultas mayores, por considerar que son parte de la amplia población vulnerable del país, lo cual fue argumentado en la primera parte del estudio.

En cuanto a la metódica, a mediados del mes de junio 2020, se diseñó y envió un guión de preguntas abiertas a los docentes universitarios eméritos de la Universidad del Zulia, utilizando plataformas virtuales tales como: correo electrónico, WhatsApp y Telegram, debido a la imposibilidad de realizar los encuentros y entrevistas de manera presencial, por la situación de las medidas preventivas, por medio del cual se pudo tener acceso a la información focalizada sobre la experiencia vivida durante la cuarentena social establecida en el país, en el contexto de la pandemia del coronavirus-COVID-19. El guión estuvo disponible durante dos semanas para ser respondido. Luego, mediante las aplicaciones WhatsApp y Telegram, se realizaron llamadas para establecer diálogos y dar a las participantes la oportunidad de manifestar o expresar ideas, sentimientos, sensaciones y pensamientos que no les fueron posible expresar de manera espontánea y extensa a través del instrumento de recolección de información.

La muestra fue por conveniencia (Battaglia, citado por: Hernández et al., 2018). Se recibió un total de 20 respuestas al guión, de los cuales fueron excluidos los extemporáneos y los que al ser revisados respondieron menos del 70\% del total de preguntas, aun cuando se intentó establecer contacto con ellos para aumentar el número de participantes. Quedando en definitiva 5 informantes clave (IC). Es importante destacar que, debido a la naturaleza de este estudio y su diseño, no se pretende efectuar una generalización ni extrapolación de los resultados obtenidos, sino "conocer la subjetividad" (Álvarez-Gayou, 2005) y comprender el sentido y significado de la experiencia vivida por los participantes. Se incorporaron como instrumentos de registro de información: el archivo Word, la laptop, el internet, el teléfono móvil, la aplicación WhatsApp y Telegram.

En resumen, la metódica consistió en: - contactar a los IC e invitarlas a participar en la investigación; envío del guión de preguntas abiertas por correo electrónico, WhatsApp y Telegram; recepción del guión por parte de los docentes; - la apropiación del texto escrito; la 
REVISTA DE LA UNIVERSIDAD DEL ZULIA. 3a época. Año $12 \mathrm{~N}^{\circ} 34,2021$ Eglee Vargas-Acosta et al. /// Cuarentena social en el contexto del COVID-19: una mirada... 442-468 DOI: http://dx.doi.org/10.46925//rdluz.34.25

construcción del texto escrito; revisión de la puntuación (dotación de sentido); elaboración de tabla con cada pregunta y la información o data de cada una de las IC; enumeración de las líneas de los párrafos del texto; lectura, relectura y subrayado del texto con colores distintivos, comparación constante: ir y volver para observar, captar y subrayar los hallazgos significativos en la comparación: palabras clave o expresiones recurrentes o relevantes y palabras elocuentes (Gurdian-Fernandez, 2007), frases explicativas, similitudes, diferencias en lo expresado por cada IC; tematización; categorización; elaboración de esquemas, matrices y gráficos para la organización de la información y codificación en temas, categorías, subcategorizas y propiedades; selección de palabras textuales (interlocutores) con mayor recurrencia; selección de palabras textuales con mayor elocuencia; y, por último, la construcción interpretativa de los significados expresados por las IC.

En el análisis de los datos e información desde la perspectiva fenomenológica, se pretende buscar la estructura y esencia de los significados posibles que los IC le atribuyen a la experiencia. Es un proceso como se dijo anteriormente de lectura, reflexión, relectura, escritura y reescritura, de manera que las investigadoras transformarán la experiencia vivida en una expresión textual, mediante la selección de palabras o frases que describan las particularidades sobre los significados, respecto a las experiencias vividas, durante la cuarentena social en el contexto de la pandemia por el COVID-19 en Venezuela.

\section{Hallazgos y análisis}

Con base en los datos recogidos, emergieron dos temas que se expresan en la Matriz l: Contexto de la Cuarentena Social; y la Matriz 2: Experiencias vividas en la cuarentana social; ambas con sus respectivas categorías, subcategorías y las palabras textuales con mayor recurrencia y elocuencia expresadas por las informantes clave.

En la matriz 1, surgió la categoría "La cuarentena social en Venezuela es diferente a la de otros países", y comprende las subcategorías: la pandemia le cayó como "anillo al dedo a este gobierno", crisis económica, crisis del sector salud, crisis de los servicios públicos y necesidad de prevención. 
REVISTA DE LA UNIVERSIDAD DEL ZULIA. 3a época. Año $12 \mathrm{~N}^{\circ} 34,2021$ Eglee Vargas-Acosta et al. /// Cuarentena social en el contexto del COVID-19: una mirada... 442-468 DOI: http://dx.doi.org/10.46925//rdluz.34.25

La subcategoría: La pandemia le cayó como "anillo al dedo a este gobierno", se expresa en palabras recurrentes, como "El país está enfrentando muchas limitaciones", "En este país..., donde no hay gasolina". Las IC, señalan respecto a la implementación de la cuarentena: "no por los casos de Coronavirus, sino por escasez de la gasolina". Desde la perspectiva de las informantes clave, esta situación "hará colapsar a todo el país" y, "La cuarentena se extenderá mes tras mes..., mientras no tengamos gasolina" ya que, es "La mejor excusa, una pandemia, para mantener a la gente en sus casas..., evitar un estallido social". Consideran que, en esta coyuntura, se está "sin visualizar una respuesta coherente y efectiva de parte del gobierno".

En el análisis se observaron diversos significados que emergieron sobre la percepción respecto al gobierno venezolano. Obsérvese esto en las palabras elocuentes: "Es un Gobierno que nos ha destrozado nuestra calidad de vida, en los términos de felicidad y de satisfacción de nuestras necesidades de vida", que "nos mantendrá así, por mucho tiempo, es decir en cuarentena". A decir de las IC, solo "para impedir que la ciudadanía salga a las calles a protestar..." "Significó para el régimen una posibilidad de mantener a flote su deteriorado sistema de gobierno. Es por todo este significado que señalan que, aun cuando la cuarentena resulta necesaria, "le cayó como anillo al dedo".

De manera que la cuarenta social ha afectado la credibilidad en el gobierno, y es expresada en la desconfianza en cuanto a la motivación para el establecimiento de la cuarentena social en el país, la cual fue percibida -a la fecha de la investigación-, como una decisión apresurada y asumida como una imposición del gobierno, es decir, como una excusa. En este sentido, para las IC, la pandemia representó para el gobierno "una posibilidad de mantener a flote su deteriorado sistema de gobierno". Según Álvarez (2020), en este contexto de pandemia el gobierno tomó medidas de emergencia y suspendió las garantías constitucionales fundamentales, también como una estrategia para el control sobre la población, es decir, el control social mediante las restricciones establecidas, como por ejemplo "QUEDATE EN CASA", es decir, mantener a las persona en el hogar, "para impedir que la ciudadanía salga a las calles a protestar", especialmente cuando "NO HAY GASOLINA" en el país; es una manera de "evitar un estallido social". 


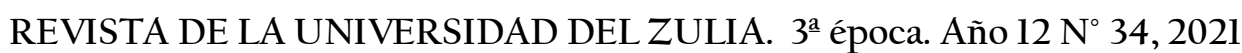

Eglee Vargas-Acosta et al. /// Cuarentena social en el contexto del COVID-19: una mirada... 442-468 DOI: http://dx.doi.org/10.46925//rdluz.34.25

\section{Matriz l. Tema: Contexto de la Cuarentena Social}

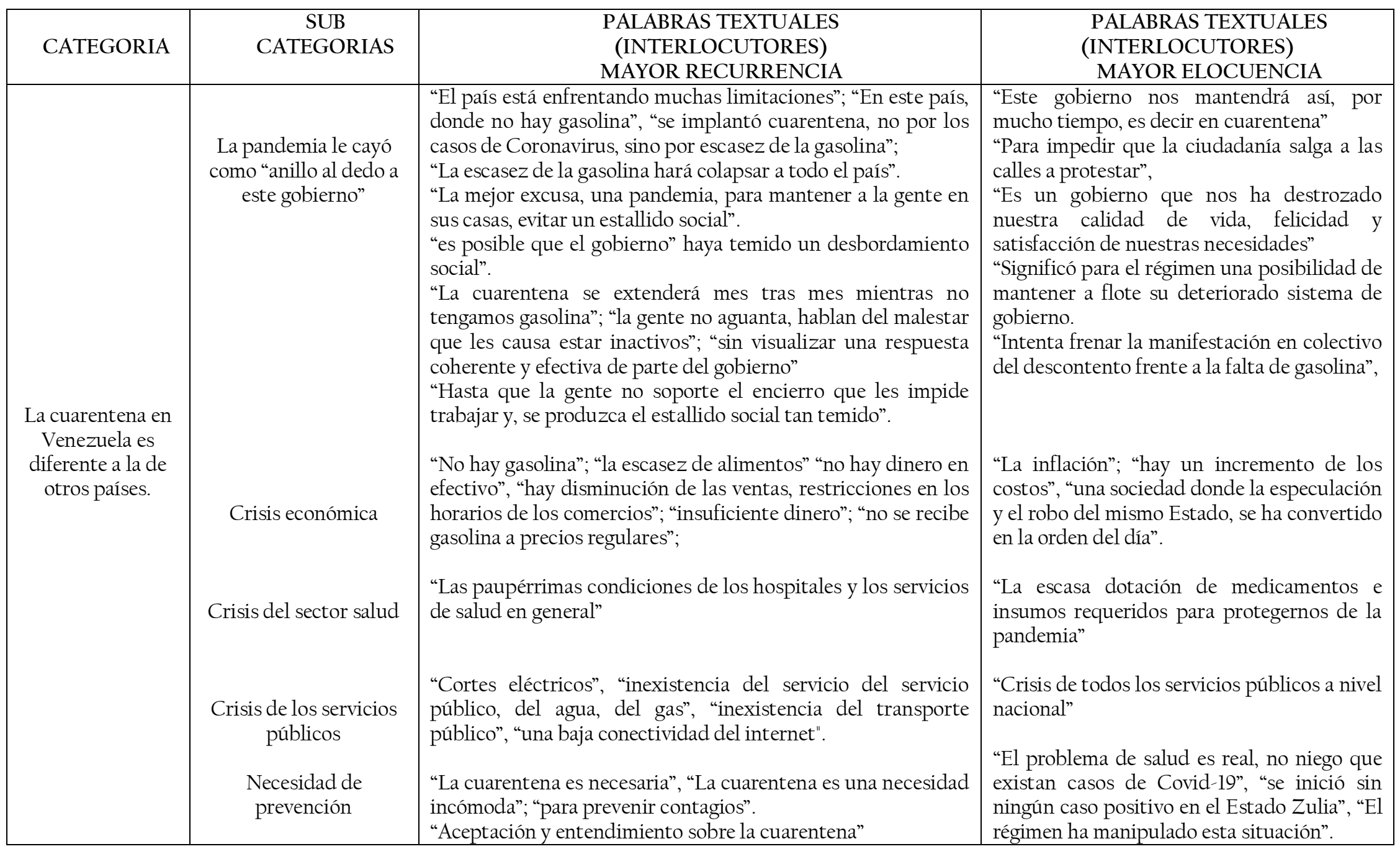


REVISTA DE LA UNIVERSIDAD DEL ZULIA. 3a época. Año $12 \mathrm{~N}^{\circ} 34,2021$ Eglee Vargas-Acosta et al. /// Cuarentena social en el contexto del COVID-19: una mirada... 442-468 DOI: http://dx.doi.org/10.46925//rdluz.34.25

También afloró una proyección "Hasta que la gente no soporte el encierro que les impide trabajar y se produzca el estallido social tan temido". Importante el planteamiento respecto a que "No hay señales de levantamiento progresivo del confinamiento, pese a que millones de venezolanos se ven forzados a romperlo para ganarse la vida en la calle, en medio de una severa crisis, con seis años de recesión, hiperinflación y el colapso de servicios como agua y electricidad" (Parra, 2020).

En cuanto a la subcategoría crisis económica, se observó como una constante expresiones que aluden a aspectos que afectan tanto el entorno individual como el colectivo; la recurrencia de palabras en cuanto a "no hay gasolina" en el país; "la escasez de alimentos", "no hay dinero en efectivo", "hay disminución de las ventas y restricciones en los horarios de los comercios"; "insuficiente dinero"; "no se recibe gasolina a precios regulares"; y las palabras con mayor elocuencia, fueron: "La inflación", "hay un incremento de los costos", "una sociedad donde la especulación y el robo del mismo Estado, se ha convertido en la orden del día”.

Estas expresiones muestran, por un lado, que se está, a decir de Landaeta, et al., (citado por Herrera-Cuenca, 2021), ante la instalación de la crisis de Inseguridad Alimentaria, debido a la situación económica: hiperinflación y la caída en la producción de los alimentos por nombrar algunos y, por el otro, que "el gravísimo problema del combustible que impide reactivar la economía y puede condenar a los ciudadanos y las empresas a una paralización de su flujo de ingresos con efectos catastróficos" (Oliveros, citado por Parra, 2020).

Con respecto a la sub-categoría: Crisis del sector salud, las palabras con mayor recurrencia fueron: "las paupérrimas condiciones de los hospitales y los servicios de salud en general"; mientras que las de mayor elocuencia se expresaron en "la escasa dotación de medicamentos e insumos requeridos para protegernos de la pandemia".

Es importante destacar que en el país, la crisis de este sector ha sido de larga data, es decir, desde finales del siglo pasado, pero se ha profundizado desde hace más de diez años con un marcado deterioro del subsistema nacional de salud en general, con la pérdida de personal sanitario debido a los bajos salarios y la migración: situación que se agudiza en los centros hospitalarios públicos, que ya presentan un franco deterioro en la infraestructura y fallas en 
REVISTA DE LA UNIVERSIDAD DEL ZULIA. 3a época. Año $12 \mathrm{~N}^{\circ} 34,2021$ Eglee Vargas-Acosta et al. /// Cuarentena social en el contexto del COVID-19: una mirada... 442-468 DOI: http://dx.doi.org/10.46925//rdluz.34.25

cuanto a los servicios básicos como el agua, electricidad, así como la falta de dotación oportuna de insumos médicos y medicamentos, déficit de camas (Richter y Stephany, 2021; Zelde et al, 2020; Altuve, 2020). Se destaca, además, el llamado "silencio epidemiológico", es decir, la no publicación del Boletín Epidemiológico del Ministerio de Salud, lo cual no contribuye a la toma de decisiones oportunas sobre la prevención de la salud en la población (Altuve, 2020).

Ante la descripción de la crisis crónica del sistema de salud en el país, puede decirse que, no existen condiciones sanitarias adecuadas para atender los casos que se han estado presentado, ni para disminuir el riesgo de contagio en la población por la pandemia del COVID-19 y, aun cuando el gobierno dispuso a nivel nacional de 46 hospitales tipo IV denominados centinelas para atender los posibles casos, "no todos los establecimientos de salud cuentan con suministros de insumos ni cupos suficientes para tratar a la población afectada" (ONG Médicos Unidos, citado por Altuve, 2020).

En la sub-categoría Crisis de los servicios públicos, se puede observar la recurrencia de frases alusivas a ésta, referidas a los cortes eléctricos, deterioro creciente del servicio de electricidad (en el caso del estado Zulia, son horas prolongadas de cortes eléctricos y múltiples fluctuaciones de la electricidad; también en cuanto al suministro de agua potable o su inexistencia. Al respecto, señalan Zelde, et al (2020) que hay un colapso de las instituciones y, por lo consiguiente de los servicios que prestan. Estos servicios, son fundamentales en la aplicación y efectividad de las estrategias prioritarias de prevención, como son las medidas de higiene, especialmente las de "lavarse las manos", para minimizar el contagio del COVID-19 (OMS, 2020).

Otras palabras recurrentes se refieren a la ineficiencia en el servicio de gas doméstico, así como del transporte público para la movilización y traslados. En cuanto a este último, es importante destacar que "la gasolina pesa muchísimo..., si hoy el gobierno decidiese relajar la cuarentena, la gente encontraría que no puede ir a trabajar... no hay cómo movilizarse (García, citado por Parra, 2020). A la fecha esta situación aún persiste.

En cuanto a palabras con mayor elocuencia, las IC señalan una "crisis de todos los servicios públicos a nivel nacional", en la que se incluye la conexión a internet, caracterizada 
REVISTA DE LA UNIVERSIDAD DEL ZULIA. 3a época. Año $12 \mathrm{~N}^{\circ} 34,2021$ Eglee Vargas-Acosta et al. /// Cuarentena social en el contexto del COVID-19: una mirada... 442-468 DOI: http://dx.doi.org/10.46925//rdluz.34.25

por "una baja conectividad". Este servicio es importante no solo en la dinámica personal y familiar, sino también en lo colectivo, ya que, para dar cumplimiento al lineamiento emanado del Estado (Presidencia de la República Bolivariana de Venezuela. 2020), respecto a la continuidad de la educación por el cierre de las instituciones educativas, es necesario para implementar la modalidad on line.

Ahora bien, con la baja conectividad del internet (Monroy, 2020) es y ha sido imposible que el proceso educativo pueda adelantarse de una manera eficaz y eficiente en la búsqueda del logro de los objetivos educativos y de la excelencia académica, que siempre ha destacado en los planes de estudios en el país. A esto se suman los significados que emergieron, sobre los niveles de estrés que se ha generado cuando las IC les ha correspondido el papel como docente contratada, o como cursantes de estudios de actualización, sin contar con este servicio.

Respecto a la subcategoría Necesidad de prevención, desde la perspectiva de las IC, la cuarentena es aceptada y entendida como "necesaria para la prevención del contagio masivo". Sin embargo, consideran que esta se inició sin que en el estado Zulia hubiese un caso positivo. Algunas expresiones más elocuentes, son: "El problema de salud es real, no niego que existan casos de Covid-19", "se inició sin ningún caso positivo en el Estado Zulia". Se muestra la pérdida de la credibilidad con respecto al gobierno, cuando señalan que "El régimen ha manipulado esta situación".

En este contexto, la categoría la cuarenta en Venezuela es diferente a la de otros países, parece estar ajustada al planteamiento referido a que "mientras en otros países conjugan el interés por la salud de la gente con el de la salud de la economía, en Venezuela la respuesta parece limitarse a prolongar la cuarentena y seguir dando la espalda a la economía" (Rossen, 2020).

También es importante en el análisis tener en cuenta la entrevista realizada a Müller autora del libro Pandemia: virus y miedos en el cual hace referencia a las reacciones de indiferencia o la negación inicial ante ésta hasta el posterior terror o estallido de pánico-, quien destaca que no se puede ver la salud y la política como campos separados, ya que la toma de decisiones con respecto a la primera "son netamente políticas. Tiene que ver con las medidas que toman los 


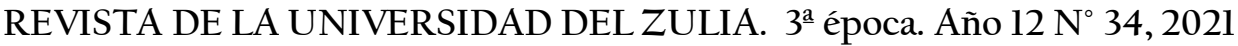
Eglee Vargas-Acosta et al. /// Cuarentena social en el contexto del COVID-19: una mirada... 442-468 DOI: http://dx.doi.org/10.46925//rdluz.34.25

Estados, con la comunicación que dan, con la reacción de las poblaciones, con su idiosincrasia, con el estado del sistema de salud" (Müller, citado por López, 2020).

Seguidamente, se presenta la Matriz 2: Experiencias vividas en la cuarentena, en la cual emergió la categoría: Afectación de la cuarentana social establecida en el contexto de la pandemia por el COVID-19, que se expresó en las subcategorías: En lo emocional, Contexto familiar y social, contexto económico, actividades laborales y afrontamiento ante la adversidad.

En la subcategoría en lo emocional, la cuarentena ha afectado las emociones que afloraron expresadas en las palabras recurrentes como: los sentimientos de miedo, tristeza, ansiedad, estrés, insomnio, mal humor, "el sentir que se está presa en el hogar", "frenada", "sienten que están ante la paralización de la vida en lo cotidiano". Dijeron experimentar "incertidumbre", por un lado, respecto a la duración de la cuarentena, la situación económica y la escasez de recursos para dar respuesta a las necesidades básicas familiares y, por el otro, en relación a la afectación física y emocional, así como a las inquietudes de iqué hacer en el caso de contagiarse? Sobre todo, en el contexto de crisis del sector salud y la economía del país, ya descrito.

Otro aspecto que llama la atención, son las expresiones como "el sentir que la vida se ha paralizado, el sentir que se está "sin vivir" (...) no tener libertad". Estos síntomas que afloran en las vivencias de las IC durante la cuarentena social, coinciden con el planteamiento de la OPS (2020), Ron, et al (2020), Khan et al (2020), Ozamiz-Etxebarria, et al (2020) y Acosta (2021), en cuanto a los impactos negativos en la salud mental de la población en general y, en lo particular en los adultos mayores, es decir, que se pueden manifestar ante la situación de incertidumbre que genera el contexto de pandemia y, más aún en el contexto de crisis crónica que atraviesa el país en los últimos años (Vargas-Acosta, 2018). 


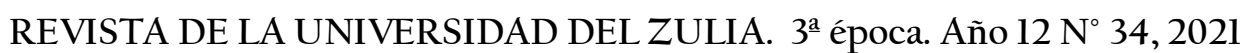

Matriz 2. Experiencias Vivenciales

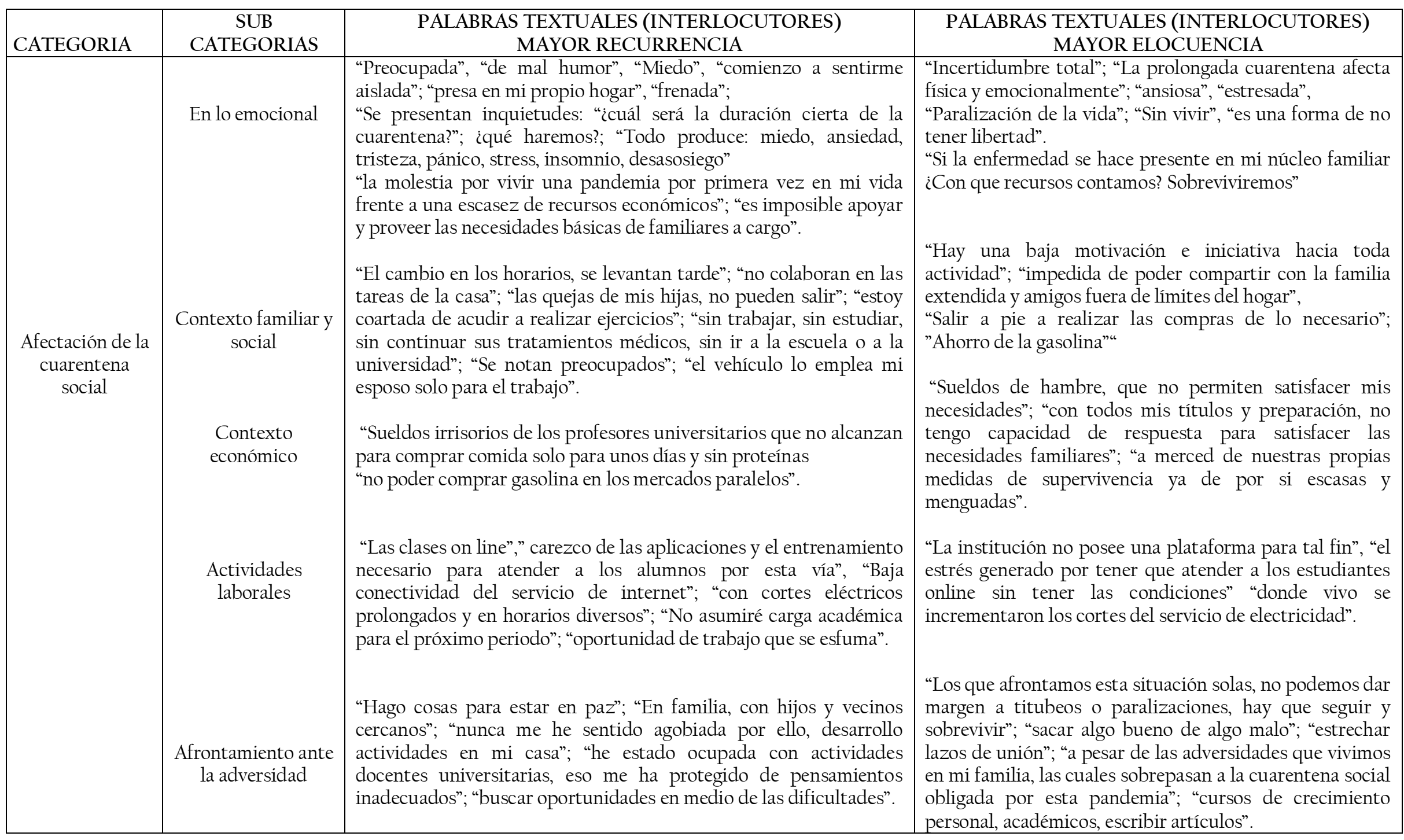


En la subcategoría contexto familiar y social, las palabras recurrentes están relacionadas con los cambios y alteraciones en la dinámica familiar, como, por ejemplo: los horarios de levantarse, la poca colaboración con las tareas en el hogar por parte de los hijos, hijas o nietos; también en cuanto a la paralización de las actividades laborales, estudios, el cumplimiento de los tratamientos médicos. En cuanto a las palabras elocuentes, manifiestan un bajo nivel de motivación e iniciativa hacia cualquier actividad.

En relación con la sub-categoría contexto económico, en los significados construidos emergieron las palabras recurrentes: "sueldos irrisorios de los profesores universitarios que no alcanzan para comprar comida solo para unos días y sin proteínas", y el no poder adquirir "gasolina en los mercados paralelos o legales"; mientras que las expresiones elocuentes precisaron tener: "sueldos de hambre, que no permiten satisfacer mis necesidades", la incapacidad de poder dar respuestas a las necesidades de la familia a pesar de contar "con todos mis títulos y preparación (...)", "a merced de nuestras propias medidas de supervivencia ya de por si escasas y menguadas".

Esto coincide con lo planteado por las investigadoras Richter y Stephany (2021: 8) en cuanto a "los salarios de hambre y la pérdida de los beneficios económicos y sociales (...)" de los trabajadores en general; y con Melean y Contreras (2020), respecto a la situación general en la que transitan los docentes universitarios en Venezuela, quienes viven las circunstancias producto de la crisis profunda económica del país, que como se ha señalado presenta una hiperinflación en la que "el 94\% de los venezolanos vive en la pobreza y aproximadamente el $30 \%$ no puede poner suficientes alimentos sobre la mesa para cumplir con los requisitos mínimos de nutrición" (Zelde, et al, 2020: 654).

Se desprende de los textos, por un lado, la desvalorización tanto de la actividad docente como de la meritocracia por parte de los que dirigen el Estado y, por el otro, las implicaciones individuales como familiares al no tener las docentes eméritas, las condiciones para dar respuestas a las necesidades básicas familiares. Cabe destacar que, según información no oficial, para el mes de febrero 2021, la canasta alimentaria se ubicó en Bs. 535.756.684,06 
(equivalente a \$281,94) (Cendas-FVM, cp. Richter y Stephany, 2021); estas investigadoras enfatizan que el salario mínimo y cesta ticket no superan los $\$ 4$ mensuales de un trabajador.

Es importante señalar que la pandemia impone alteraciones en las condiciones económicas en los hogares de las docentes eméritas; estos son efectos socio-económicos, al no recibir sueldos acordes a los méritos académicos obtenidos durante su trayectoria, es decir, que la afectación de la cuarentena ha trascendido de lo individual-emocional-familiar a lo socialcolectivo.

En cuanto a la sub-categoría actividades laborales, expresaron de manera recurrente el no contar con las innovaciones tecnológicas necesarias, ni el entrenamiento para poder viabilizar las clases on line; además es persistente el aspecto sobre la baja conectividad del servicio del internet, los cortes eléctricos que afecta una óptima y adecuada comunicación con los estudiantes (Cardozo y Guevara, 2020). Es importante resaltar que, estos significados emergieron en la matriz anterior respecto a la crisis de los servicios públicos y que generan cierta inestabilidad en las profesoras -que aun siendo eméritas, siguen desempeñándose como docentes- poniendo en riesgo, inclusive la continuidad de su relación de trabajo (Serrano, 2021), que se manifiesta en el siguiente texto: "No asumiré carga académica para el próximo período", o ante alguna "oportunidad de trabajo que se esfuma" por el contexto descrito.

Esta sub-categoría comprende palabras elocuentes, tales como la inexistencia de una plataforma por parte de las instituciones de educación superior para viabilizar la modalidad on line. Otro aspecto a destacar ha sido que al tener que implementar esta modalidad desde el hogar, se ha generado en las IC, el estado de estrés debido a que no tienen las condiciones para ello.

En el marco de lo anteriormente señalado, se destaca que en el cumplimiento del lineamiento sobre el cambio brusco de la modalidad presencial a la modalidad on line, es importante señalar los siguientes elementos involucrados: a) las carreras profesionales en las universidades del país han sido concebidas bajo la modalidad presencial; b) las instituciones universitarias no cuentan con la infraestructura y plataforma para construirle viabilidad a la implementación de esta modalidad; c) los profesores no tienen la formación necesaria para 
liderar este tipo de proceso de formación como tampoco los recursos: internet, teléfonos de nueva generación, equipos de computadores, entre otros; d) todo esto genera en los docentes niveles de estrés por no poder cumplir con sus labores académicas como trabajo o como participantes en estudios de actualización o formación de postgrado.

En la subcategoría Afrontamiento ante la adversidad, emergieron significados tales como: "estar unidos con la familia y los vecinos", "hacer actividades universitarias o del hogar, para no sentirse agobiada" o "protegerse de pensamientos no favorables"; también resalta la "búsqueda de oportunidades a pesar de las dificultades". En cuanto a las palabras elocuentes se precisaron “(...) hay que seguir y sobrevivir", "la búsqueda de lo bueno ante la adversidad", "la realización de cursos académicos y de crecimiento personal", entre otros. Se observan iniciativas para sobreponerse a las limitaciones propias de la cuarentena y el confinamiento social.

Para cerrar este análisis, puede observarse que en Venezuela la pandemia representa un problema que "tiene una dimensión universal y afecta valores tan importantes como la vida misma, la libertad, la actividad humana, la relación interpersonal, la generación de bienes y servicios para la supervivencia" (Roosen, 2020). Este autor destaca que el gobierno ha anunciado tener la pandemia bajo control y, de manera lineal ha prolongado la cuarenta entre una semana flexible y otra semana restringida, con "limitaciones de movilidad ciudadana, el cierre de las fronteras, algún aprovisionamiento de material médico sería suficiente para su concepto de seguridad. Incluso las carencias como la falta de gasolina o la escasísima disponibilidad monetaria jugarían a su favor. ¿Por cuánto tiempo?" (Roosen, 2020). Es decir, que la pandemia se da en el país bajo unas condiciones sociales y económicas críticas y difíciles para la sociedad en general y, en particular para la población vulnerable, como las docentes universitarias eméritas, los adultos mayores, entre otros.

\section{Conclusiones}

Con base al proceso analítico realizado desde la perspectiva de esta investigación, los significados construidos por las docentes universitarias eméritas de la Universidad del Zulia- 
Venezuela, con relación a las experiencias vividas en la cuarentena social en el contexto de la pandemia por el COVID-19, se organizaron mediante temas, categorías y subcategorías.

El tema referido a "el contexto de la cuarentena social, se expresó mediante la categoría " $\mathrm{La}$ cuarentena en Venezuela, es diferente a la de otros países". En este sentido, en los datos suministrados por las IC, emergieron significados con relación a las características actuales de Venezuela y las condiciones de la crisis crónica económica, política y social que se vive en cada uno de los sectores de la vida nacional, impactándolos de manera negativa, con mayor énfasis en el sistema de salud y en los servicios públicos, esenciales para cumplir con las medidas preventivas establecidas por la OMS en el marco de la pandemia por COVID-19.

Otros significados construidos que emergieron en la data es el factor de la provisión del combustible (gasolina) y las consecuencias que conlleva en la paralización del sector productivo, el comercio en general, la movilización de la población para abastecerse con los alimentos, medicinas, entre otros. También sobresale el elemento político, vinculado a la pérdida de credibilidad en el gobierno por parte de las IC, asociado a la percepción que tienen respecto a la toma de decisiones con el establecimiento de la cuarentena social en el país.

El segundo tema que emergió fue las "Experiencias vividas en la cuarentena", reflejada en la categoría: "Afectación de la cuarentana social", que permitió visualizar el impacto en diversas dimensiones; una de ellas en lo emocional, que se evidenció en los cambios experimentados en los sentimientos y estados anímicos, como el estrés, preocupaciones, tristeza, mal humor, ansiedad, miedo, angustia, insomnio, desasosiego, aislamiento y pánico, entre otros. También se generaron cambios en el desenvolvimiento de la vida cotidiana del núcleo familiar y social.

Otros significados se refieren a la economía familiar y a las actividades laborales. La primera está vinculada a la no satisfacción de las necesidades básicas de subsistencia de los miembros de la familia; situación que contribuye a elevar los niveles de estrés y ansiedad, toda vez que los sueldos que reciben en su actividad como docentes universitarias eméritas, no están acorde con los méritos académicos logrados durante la trayectoria laboral, ni con la hiperinflación que vive el país y, por ende, con el costo de la canasta básica alimentaria. La segunda, referida a la realización de actividades laborales académica en condición de 
contratada, para lo cual en el contexto pandemia, se exige la implementación de "la modalidad de clases on line", y ante las condiciones críticas de los servicios de electricidad, baja conectividad del internet, equipos obsoletos y la débil formación en el manejo de las innovaciones tecnológicas para el proceso de aprendizaje, generan una mayor complejidad que elevan los niveles de estrés ante el compromiso y responsabilidad en el trabajo a realizar.

Finalmente, con base en los significados construidos por las IC, y desde la perspectiva de las investigadoras, puede decirse que la categoría central emergente "La cuarentena en Venezuela es diferente a la de otros países" en el contexto de la pandemia por COVID-19, es de interés conceptual para la comprensión del fenómeno estudiado, ya que reflejó la superposición de dos condiciones: la crisis humanitaria compleja y la pandemia, teniendo un impacto multidimensional negativo, que afectó además de las ya deterioradas condiciones materiales de vida, lo socio-emocional y valores como la libertad, la paz, la convivencia intrafamiliar, entre otros.

Los resultados de esta investigación arrojan luces que pueden servir de sustento para el diseño de estrategias de intervención social, dirigidas a las personas y familias en condiciones de vulnerabilidad en el contexto ya descrito, contribuyendo así a la mitigación de los impactos en la calidad de vida de las personas afectadas.

\section{Referencias}

Acosta, Yorelis J. (2021). La salud mental en Venezuela a un año del inicio de la pandemia. En: Dossier Venezuela: Emergencia en salud y pandemia. Delgado B., Andy (Coordinadora). Revista Cuadernos del Cendes Año 38, № 106. Editada por el Centro de Estudios del Desarrollo, Cendes. Universidad Central de Venezuela. Disponible en: http://www.ucv.ve/cendes Recuperado: 13/08/2021.

Álvarez-Gayou, J. (2005). Cómo hacer investigación cualitativa. Fundamentos y Metodología. Paidós, Editorial. México. P. 222

Altuve, A. (28 de marzo de 2020). "Desde Venezuela: una pandemia en medio de la crisis". Salud con Lupa. [En línea]. Disponible en: https://saludconlupa.com/noticias/desde-venezuelauna-pandemia-en-medio-de-la-crisis/ Recuperado: 05/05/2020 
Broche-Pérez, Y., Fernández-Castillo, E., Reyes L., D. (2020). “Consecuencias psicológicas de la cuarentena y el aislamiento social durante la pandemia de COVID-19". Revista Cubana de Salud Pública. 2020; 46 (Supl. especial): e2488. [En línea]. Disponible en: https://www.scielosp.org/pdf/rcsp/2020.v46suppll/e2488/es Recuperado: 05/08/2020.

Cardozo, Rosa, Guevara, Harold y Ortunio Magaly (2020). El docente universitario venezolano: Calidad de vida y migración. Rev Bioet Latinoam; vol. 24: 120-131 Disponible en: https://revistas.udec.cl/index.php/paideia/article/download/3349/3413/6934

Carrillo R., Alejandra (2018). Sistema de salud en Venezuela: iun paciente sin remedio? Cuaderno de Saúde Pública. Reports in Public Health 34 (3): 56-62. Disponible en: https://doi.org/10.1590/0102-311X00058517 Recuperado: 12/08/2021.

Comisión Económica para América Latina y el Caribe (CEPAL). Panorama Social de América Latina 2020. (LC/PUB.2021/2-P/Rev.l) Santiago, Chile, 2021.

Daniels Joe P. (2020). Venezuelan migrants "struggling to survive" amid COVID-19. World Report (395) Lancet. Mar 28. Disponible en: https://doi.org/10.1016/S0140-6736(20)30718-2 Recuperado: 12/08/2021.

García, A. y Cuéllar-Flores I. (2020). "Impacto Psicológico del confinamiento en población infantil y como mitigar sus efectos: Revisión Rápida de la evidencia". [En línea]. Doi: https://doi.org/10.1016/j.anpedi.2020.04.015 Recuperado: 24/05/2020

Gurdián-Fernández Alicia (2010). El Paradigma Cualitativo en la Investigación SocioEducativa. Editorial Coordinación Educativa y Cultural Centroamericana (CECC). Agencia Española de Cooperación Internacional (AECI). Re-edición. Costa Rica. P-283. Disponible en: http://repositorio.inie.ucr.ac.cr/bitstream/123456789/393/1/paradigama\%20cualitativo.pdf

Recuperado: 05/05/2019

Hernández, R., Fernández, C., Baptista, P. (2018). Metodología de la Investigación. Mc Graw Hill. 6ta edición.

Herrera-Cuenca, Marianella (2021) Aspectos cualitativos de la Inseguridad Alimentaria dentro de la crisis de salud venezolana. Pp-53-77. En: Dossier Venezuela: Emergencia en salud y pandemia. Delgado B., Andy (Coordinadora). Revista Cuadernos del CENDES. Año 38, № 106. Editada por el Centro de Estudios del Desarrollo, CENDES. Universidad Central de Venezuela. Disponible en: http://www.ucv.ve/cendes Recuperado: 13/08/2021. 
Khan, S., Siddique, R., Li, H., Ali, A., Shereen, M. A., Bashir, N., y Xue, M. (2020). Impact of coronavirus outbreak on psychological health. Journal of Global Health, 10 (1), 1-6. Disponible en: https://doi.org/10.7189/jogh.10.010331 Recuperado: 14/08/2021.

López, M. (2020). Mónica Müller: "La salud es política, no son dos campos separados". Saludconlupa. Una dosis de periodismo con calidad. Entrevista realizada el 17/04/2020. [En línea]. Disponible en: https://saludconlupa.com/entrevistas/monica-muller-la-salud-espolitica-no-son-dos-campos-separados/ Recuperado: 12/03/2021.

Martínez-Miguélez, Miguel (2006). Ciencia y arte en la metodología cualitativa. MéxicoMéxico DF. Editorial Trillas.

Melean R., Rosana, Contreras B., José (2020). Universidad venezolana en tiempos en pandemia: ¿Acción O Reacción? Ante la nueva normalidad. Utopía y Praxis Latinoamericana. Vol. 25, número. Esp. 13, pp. 194-209

Monroy, Fran (2020) “Conectividad en Venezuela no soporta más exigencias”. En: Periódico digital Tal Cual. 17/04/2020. [En línea]. Disponible en: https:/talcualdigital.com/conectividaden-venezuela-no-soporta-mas-exigencias/ Recuperado: 10/12/2020.

Organización Mundial de la Salud (2021) WHO coronavirus (COVID-19) Dashboard. [En línea] Disponible en: https:/covid19.who.int/ Recuperación: 18/07/2021

Organización Mundial de la Salud (2021) "Coronavirus. Sinopsis, prevención y síntomas". [En

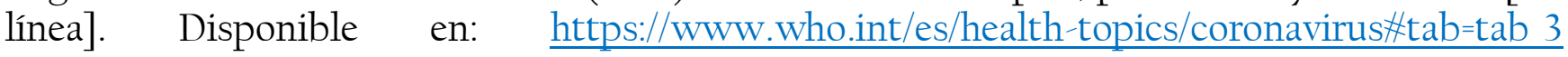
Recuperación: 18/07/2021

Organización de las Naciones Unidad (2021). "La variante Delta del COVID-19 nos ha llevado a un momento muy peligroso de la pandemia". 2/07/2021. [En línea]. Disponible en: https://news.un.org/es/story/2021/07/1493992 Recuperado: 15/07/2021

Organización Mundial de la Salud (2020). “Infecciones por coronavirus". [En línea]. Disponible en: https://www.who.int/topics/coronavirus infections/es/ Recuperado: 12/05/2020

Organización Mundial de la Salud (2020). "Consideraciones relativas a las concentraciones multitudinarias en el contexto de la COVID-19". [En línea]. Disponible en https://www.who.int/topics/coronavirus infections/es/ Recuperado: 15/05/2020

Organización Mundial de la salud (2020) "Preguntas y respuestas sobre la enfermedad por coronavirus (COVID-19)". Disponible en: https://www.who.int/es/emergencies/diseases/novelcoronavirus-2019/advice-for-public/q-a-coronaviruses Recuperado: 06/11/2020 
Organización Mundial de la salud (2020). "Panel de la OMS sobre la enfermedad por coronavirus (COVID-19)" [En línea]. Disponible en: https://covid19.who.int/table Recuperado: 29/07/2020

Organización Mundial de la salud. (2020). "Alerta Epidemiológica Oleadas y brotes recurrentes de COVID-19". 9 de octubre de 2020. [En línea]. Disponible en: http://www.paho.org/ Recuperado 12/11/2020

Organización Panamericana de la Salud (2021). Daily COVID-19 Update: 16 July 2021. [En línea]. Disponible en: https://www.paho.org/en/documents/paho-daily-covid-19-update-16july-2021 Recuperado: 18/07/2021

Organización Panamericana de la salud (2020). "Salud Mental y COVID-19". [En línea]. Disponible en: https://www.paho.org/es/salud-mental-covid-19 Recuperado 21/06/2020

Ortiz, A. (2015). Enfoques y métodos de investigación en las Ciencias Sociales. Ediciones de la U. Colombia.

Ozamiz-Etxebarria, Naiara, Dosil-Santamaría, María, Picaza-Gorrochategui, Maitane y Idoiaga-Mondragon, Nahia. (2020) Stress, anxiety and depression levels in the initial stage of the COVID-19 outbreak in a population sample in the northern Spain. Cad Saude Publica. 36 (4), 1-9. Disponible en: https://doi.org/10.1590/0102-311X00054020

Parra, F. (2020). “Cuarentena en Venezuela: iconfinamiento obligado por escasez de gasolina?”. [En línea]. Disponible en: https:/elnacional.com/venezuela/cuarentena-envenezuelaconfinamiento-obligado-por-escasez-de-gasolina/ Recuperado 20/05/2020

Presidencia de la República Bolivariana de Venezuela (2020). Gaceta Oficial de la República Bolivariana de Venezuela No 6.519 Extraordinario. Viernes 13 de marzo de 2020. Año CXLVII MES VI Caracas. [En línea]. Disponible en: http://www.unes.edu.ve/index.php/tag/ministeriode-poder-popular-para-educacion-universitaria-ciencia-y-tecnologia/ 20/06/2020

Richter, Jacqueline y Stephany, Keta (2021). Derechos laborales y acción colectiva. El sector salud venezolano en pandemia. En: Dossier Venezuela: Emergencia en salud y pandemia. Delgado B., Andy (Coordinadora). Revista Cuadernos del Cendes Año 38, № 106. Centro de Estudios del Desarrollo, Cendes. Universidad Central de Venezuela. Disponible en: http://www.ucv.ve/cendes Recuperado: 15/08/2021 
REVISTA DE LA UNIVERSIDAD DEL ZULIA. 3ㄹ época. Año 12 N³4, 2021 Eglee Vargas-Acosta et al. /// Cuarentena social en el contexto del COVID-19: una mirada... 442-468 DOI: http://dx.doi.org/10.46925//rdluz.34.25

Rodríguez, María (2008). "Emociones y salud: Algunas consideraciones". Revista PsicologiaCientifica.com, 10(5). [En línea]. Disponible en: http://www.psicologiacientifica.com/emociones-y-salud Recuperado: 20/06/2020

Roosen, Gustavo (2020). “Todo por el Control”. En: Diario El Nacional. Columna de opinión. Publicado: 18/05/2020. [En línea]. Disponible en:

https://www.elnacional.com/opinion/todo-por-el-control// Recuperado: 20/05/2020

Sánchez-Villena, A. y De La Fuente-Figuerola, V. (2020) "COVID-19: Cuarentena, Aislamiento, Distanciamiento Social y Confinamiento iSon lo mismo?". Carta al editor. Universidad Privada del Norte, Cajamarca, Perú, p. 73-74. [En línea]. Disponible en: https://www.analesdepediatria.org/es-covid-19-cuarentena-aislamiento-distanciamientosocial-articulo-S1695403320301776 Recuperado: 03/07/2020.

SWI swissinfo.ch (2021) "OMS: la pandemia está en momento "muy peligroso" por auge de variante delta". [En línea] Disponible en: https://www.swissinfo.ch/spa/coronavirusoms oms-la-pandemia-est $\%$ C3\%Al-en-momento-muy-peligroso-por-auge-de-variantedelta/46755046 Recuperado: 08/07/2021

Unión Radio (2021) "Especialista prevé mejor conexión a internet a finales del 2021 en Venezuela". 27/01/2021. [En línea]. Disponible en: https://800noticias.com/especialista-prevemejor-conexion-a-internet-a-finales-de-2021-en-venezuela Recuperado: 08/07/2021

Vargas-Acosta, E. (2018) Cultura Política Democrática en Venezuela. Tesis Doctoral presentada para optar al grado de Doctora en Ciencia Política. División de Estudios para Graduados. Facultad de Ciencias Jurídicas y Políticas. Universidad del Zulia (p.255).

Zelde Espinel, Chaskel Roberto, Berg Ryan, Flores, Hermes, Gaviria Silvia, Bernal Oscar, Berg, Kim Muñoz Carlos, Larkin Marisa y Shultz, James (2020). Venezuelan migrants in Colombia: COVID-19 and mental health. Lancet Psychiatry. Volumen 7: 653-655. Disponible en: https://www.thelancet.com/action/showPdf?pii=S2215-0366\%2820\%2930242-X

Recuperado: 18/07/2021 